\title{
Cognitive impairment and its consequences in everyday life: experiences of people with mild cognitive impairment or mild dementia and their relatives
}

Maria Johansson, Jan Marcusson and Ewa Wressle

\author{
Linköping University Post Print
}

\section{Tweet}

N.B.: When citing this work, cite the original article.

Original Publication:

Maria Johansson, Jan Marcusson and Ewa Wressle, Cognitive impairment and its consequences in everyday life: experiences of people with mild cognitive impairment or mild dementia and their relatives, 2015, International psychogeriatrics, 1-10.

http://dx.doi.org/10.1017/S1041610215000058

Copyright: Cambridge University Press (CUP): STM Journals http://www.cambridge.org/uk/

Postprint available at: Linköping University Electronic Press http://urn.kb.se/resolve?urn=urn:nbn:se:liu:diva-115307 
Cognitive impairment and its consequences in everyday life: experiences of people with mild cognitive impairment or mild

\section{dementia and their relatives}

Maria M Johansson, Occupational Therapist, Jan Marcusson, Professor, and Ewa

Wressle, Associate Professor

Department of Clinical and Experimental Medicine, Geriatrics, Linköping University, Linköping, Sweden

Corresponding author: Maria M. Johansson, Department of Geriatric Medicine, University Hospital, SE-581 85 Linköping, Sweden. Tel. +46 1010380 30; fax: +46 1010341 41. E-mail: maria.m.johansson@liu.se 


\section{ABSTRACT}

Background: The aim of this study was to explore experiences of cognitive impairment, its consequences in everyday life and need for support in people with mild cognitive impairment (MCI) or mild dementia and their relatives.

Methods: A qualitative approach with an explorative design with interviews was chosen. The participants included five people with MCI and eight people with mild dementia and their relatives. All participants were recruited at a geriatric memory clinic in Sweden. The Grounded Theory method was used.

Results: The following categories emerged: noticing cognitive changes; changed activity patterns; coping strategies; uncertainty about own ability and environmental reactions; support in everyday life; support from the health care system; consequences in everyday life for relatives; and support for relatives. The main findings were that people with MCI and dementia experienced cognitive changes that could be burdensome and changed activity patterns. Most of them, however, considered themselves capable of coping on their own. The relatives noticed cognitive changes and activity disruptions to a greater extent and tried to be supportive in everyday life. Degree of awareness varied and lack of awareness could lead to many problems in everyday life.

Conclusions: Perceived cognitive impairment and its consequences in everyday life were individual and differed among people with MCI or dementia and their relatives. Thus, health care professionals must listen to both people with cognitive impairment and their relatives for optimal individual care planning. Support such as education groups and day care could be more tailored towards the early stages of dementia.

Key words: Alzheimer's disease, Activities of daily living, Qualitative research 
Running title: Everyday life with MCI or mild dementia

\section{Introduction}

As life expectancy increases worldwide, so does the prevalence of dementia (Qiu et al., 2009). The proportion of older people in the Swedish population is one of the highest in the world (Larsson and Thorslund, 2006) and age is the greatest risk factor for cognitive impairment. Mild cognitive impairment (MCI) is considered as a precursor to dementia or as the boundary between normal aging and dementia (Petersen et al., 2014). The most common form of dementia is Alzheimer disease (about 50-70\%), but other dementia conditions and mixed forms exist. The number of people with dementia in Sweden has been estimated to be 140000 (SBU, 2006) and it is increasing. Globally, the prevalence of dementia is estimated to more than 25 million (Qiu et al., 2009). It is important to investigate dementia at an early stage to identify any curable conditions, deploy the right medical treatment and provide appropriate support and assistance to people with dementia and relatives.

Dementia affects functional ability. Previous research shows that instrumental activities of daily life (IADL), such as use of the telephone and public transportation, and management of medications and finances (Avlund and Fromholt, 1998; Barberger-Gateau et al., 1999) are affected by cognitive decline. These activities might be used when screening for dementia (Barberger-Gateau et al., 1992). These activities and other complex activities are often already impaired at the MCI stage (Pedrosa et al., 2010; Yeh et al., 2011). It has also been seen that people with MCI need more time to cope with IADL (Wadley et al., 2008). The Lawton scale of Instrumental Activities of Daily Living is a commonly used measure in clinical settings (Lawton and Brody, 1969). More basic activities of daily living in the Katz' Index of independence in 
Activity of Daily Living (ADL) (Katz et al., 1963), such as bathing, dressing, toileting, transferring, continence and feeding are affected in the later stages of dementia (Delva et al., 2014).

Most of the studies focusing on difficulties in everyday life are quantitative using different instruments and tests. All of these include relatives' ratings of everyday functioning. The experiences of people with dementia are less often investigated because they are seen as a less reliable source due to insight problems. Some qualitative research has emerged in the field (De Vriendt et al., 2012; Öhman et al., 2008). One study focusing on the strategies that people with dementia use to manage their difficulties showed that they use their senses (sight, hearing and touch) to compensate for declining cognition; they adapt their time and try to rely on old patterns and routines of everyday life. It has been suggested that they use these strategies to gain a sense of control over their life rather than to be as effective as before (Öhman et al., 2008). People with Alzheimer disease have been found to be aware of their disease but not able to manage the practical and cognitive implications of their impairments in everyday life. They are not always well calibrated with their relatives (spouses) regarding thoughts about the disease and problems in handling the cognitive deterioration (Mårdh et al., 2013). Another study (Frank et al., 2006) compared the experiences of people with MCI or dementia and their relatives but did not focus specifically on the impact of cognitive impairment on everyday life in terms of activity performance or on the person's experiences in terms of support. Therefore, we wanted to go further than describing people's experience of cognitive impairment and its consequences in everyday life; we also wanted to find out whether they experienced any need for support. Extended knowledge could improve clinical practice when evaluating dementia and planning for 
interventions. Thus, the aim of this study was to explore experiences of cognitive impairment, its consequences in everyday life and the need for support felt by people with MCI or mild dementia and their relatives.

\section{Methods}

A qualitative approach with an explorative inductive design with interviews was chosen. Convenience sampling was used; people with MCI or mild dementia were recruited at a geriatric memory clinic. A heterogeneous sample regarding gender, age and time since diagnosis was chosen.

\section{Participants}

The participants $(n=26)$ included five people with MCI and eight people with mild dementia and their relatives (two children and 11 spouses). Diagnostic criteria from a key symposium for MCI (Winblad et al., 2004) or the DSM IV criteria for dementia (APA, 2004) were followed and the participants with an MCI or dementia diagnosis were at level 3 or 4 according to the Global Deterioration Scale (Reisberg et al., 1982). Eight were on dementia medication. Seven of the eight people with dementia had Alzheimer disease and one had mixed dementia. The characteristics of the participants are presented in Table 1.

\section{Recruitment and procedures}

The participants were first contacted by a nurse who was involved in their investigation and treatment at the clinic. They were given information about the study either by phone or during a regular visit to the clinic. If the person agreed, the interviewer (MJ), an occupational therapist at the memory clinic with long experience of clients with MCI and 
dementia, contacted the person by phone to provide more information about the study. The interviewer was not involved in the person's investigation or treatment at the clinic. Those who agreed to participate were sent written information together with a time schedule for the interview. If the person accepted, a relative was contacted by phone. Informed written consent was obtained from all participants. Ethical approval was granted by the Regional Ethical Review Board in Linköping (Dnr: 2012/387-31). The interviews were conducted at the memory clinic or in the participant's home according to their choice. An interview guide with topics covering different occupational areas (work, leisure, social participation, relations and communication, household activities and personal activities) and need for support was used. The purpose of the guide was to generate conversation and questions were added if and when new areas of interest arose. All participants were interviewed separately except for one person who wanted the relative present as a support during the interview. The interviews lasted between 40 and 95 minutes and all participants were able to express themselves and were willing to share their stories. They were given time to gain trust in the interviewer both by phone and before and after the interview.

\section{Data analysis}

The interviews were recorded and transcribed verbatim by the interviewer. The analyses were performed and inspired by the Grounded Theory methodology (Strauss and Corbin, 1990). The interviews were read through repeatedly to get a thorough narrative understanding of the text; thereafter a process of open coding was applied. Codes that seemed to pertain to the same phenomena were categorized together, compared and revised. The codes were then put together in a new way as in axial coding, categorized and named (Strauss and Corbin, 1990). Data 
collection and analyses were carried out in parallel until the final categories were set and no new data emerged (saturation was reached). The first author undertook the analysis, which was then discussed with and checked by the third author (EW) to ensure the quality and credibility.

\section{Results}

The following categories emerged: noticing cognitive changes; changed activity patterns; coping strategies; uncertainty about own ability and environmental reactions; support in everyday life; support from the health care system; consequences in everyday life for relatives; and support for relatives.

The results are presented from the participant's and relatives' perspectives under each category except for the categories on consequences in everyday life for relatives and support for relatives, which only concerns relatives. The results are illustrated with quotations. The abbreviations used in the quotations are $\mathrm{P}$ for people with MCI or dementia and $\mathrm{R}$ for relative. The numbers stand for the order in which the interviews were performed (people with MCI or dementia and relatives, respectively).

\section{Noticing cognitive changes}

The cognitive changes in everyday life described by the people with MCI or dementia were mostly short-term memory problems such as remembering what they intended to do or say and remembering names. Other cognitive impairments reported included loss of initiative, difficulties with concentration, language, agnosia, fatigue, visual perception, orientation to place and sound sensitivity. Impaired cognition was sometimes perceived as very annoying and bothersome and could contribute to anger or sadness. Some people had to 
quit working because of their illness. Problems increased when routines and/or environmental factors were changed.

So they started then with lots of new computers and we were supposed to write in and do things, yeah, well I just did not get it together so well

It was common for people with MCI or dementia to refer to normal aging and some did not experience any memory impairment.

No, I've never experienced any memory impairment in everyday life, no; so I do not have any feel for it and I think then it's great if it could go on and think so yes ... I was probably a bit surprised that they got hold of me down there actually [referring to the memory clinic] (P3)

Well then, I'm getting a little ... sometimes I miss a name and stuff. But I guess with age and everything that you might start to get a little forgetful but I'm not active today, I'm not working of course and so ... (P1)

Memory impairment was also described in all cases by relatives but to a greater extent. Relatives also noticed impaired executive function, orientation in time, judgment, difficulty with seeing in context/how things belong together, and mental and psychomotor slowness.

How well the people with MCI or dementia were aware of their impaired cognition or the consequences of it differed according to the relatives. People with dementia were perceived as less aware but some people with MCI were also perceived as lacking insight by relatives. Relatives had problems with how to handle this in everyday life. Friction arose when the people with dementia did not understand the effects of their behaviour. It could be difficult to talk about the diagnosis and the consequences in everyday life. 
I know he knows in a way but he doesn't want to talk about it. We can't talk about it, he doesn't want to. (R1)

He knows that he has a disease but does not understand it, does not see his shortcomings; he believes it will come in the future. (R7)

I do wish that he would be more aware so we could discuss and plan for the future. Short-term accommodation is impossible as long as he doesn't have insight into his problems. (R1)

\section{Changed activity patterns}

When the people with MCI or dementia described their daily chores, it was revealed that various activities had become more difficult to manage and that everyday tasks took longer time to complete. Some activities had been taken over by relatives.

Those trivial daily duties take a longer time to complete for me. (P4)

I leave the use of the computer to my wife. She pays the bills and all that. (P1)

This was confirmed by relatives but to a greater extent. The people with MCI or dementia described the changes more as a natural effect of aging and a lack of interest in the chores.

People with MCI, but particularly those with dementia, tended to let leisure activities slide. Several examples of giving up or changes in hobbies or interests were indicated. This was confirmed by relatives who also gave additional examples (e.g. maintaining the summerhouse, managing the boat). Leaving home for trips or holidays had become less interesting to the people with MCI or dementia.

It is not that exiting anymore, I suppose that I could go but not for long and not in large groups.

This was seen as a bigger problem by relatives who still wanted to do these things. 
Having a dog was a great help for two people with dementia, both for making them go out on a daily basis and for social contacts meeting people on their daily walks.

Other activities that were changed included managing finances, handling medications, cooking, baking, shopping, washing and cleaning and keeping track of papers and bills.

I could not even bake when I got home because I did not know what grams and kilos were. (P7)

Relatives reported more changes with regard to home maintenance. Talking about doing things without actually doing them was common among people with dementia according to the relatives.

She has a large garden as you saw, and she can talk about weeding the garden plot but she doesn't do it, it is the same with writing Christmas cards for example. (R13)

Driving was affected in different ways. Some people were banned from driving because of their dementia. Some of these people did not really understand why but had somehow accepted the fact. People who were still driving had adapted their driving in some way (e.g. driving only shorter distances, not driving in the dark or keeping to familiar environments). Concerns about minor incidences and for how long one would be able to continue driving came up. Relatives described more problems with driving such as difficulties with orientation, alertness, attention and difficulties at roundabouts. Not being allowed to drive the grandchildren was mentioned by some relatives and in these cases it was their children who had forbidden the people with MCI or dementia to do so.

Conversation, especially in larger groups, was listed by many people as increasingly difficult. 
I was at my sister's birthday party, and then I felt that I didn't keep up with the conversations. She also told my husband afterwards that I was quiet (P9)

Reading, writing and talking on the telephone was mentioned as being difficult and use of mobile phones was limited using only basic functions such as answering and making calls. This was confirmed by relatives with the exception of writing. Watching TV programmes with a lot of characters were reported to be problematic.

I used to be good at reading manuals, but that gets worse and worse. (P9)

I can notice that when I'm reading it doesn't stick, I have to reread over and over again. (P2)

Also handling practical issues such as installing and using telephones, TV and computer devices were problematic according to relatives. Some relatives reported that personal activities such as taking care of personal hygiene and remembering to change clothes were lacking.

Relatives also reflected on the changes they saw in people with MCI or dementia and the possible effects of premorbid function, comorbidity, medication and the peoples' interests.

\section{Coping strategies}

Several examples of strategies for how people with MCI or dementia dealt with their problems in everyday life emerged. Using memory aids (outer strategies) such as taking notes in various forms, using a calendar, a dosage unit for medications, and an alarm watch were mentioned. These strategies were more common and seemed to work better for those who were aware of their memory problems. Sometimes these strategies were initiated and performed by relatives. Using cognitive strategies (inner strategies) such as trying to think systematically about how to find belongings or going back to the starting point (when forgetting what to do next, 
etc.), repetition (reading over and over again), and controlling own actions (e.g. checking the oven before leaving home, checking the bills) were also mentioned by people with MCI or dementia. Asking for a relative's help could, however, be seen as a last resort. For many it was important to maintain the structure of everyday life, taking one day at a time, and keeping to routines and familiar surroundings.

How well the strategies were working varied. For example, taking notes or doing lists was not always helpful.

I need to write things down more often now. Fill in or write down what to do and stuff ...The dentist, for example, I thought it was today but it was not until Monday and the appointment I had with you today, I thought it was half past three, but it was half past one. So everything was just wrong! I read the notes and when I called the dentist to change the time, they told me I was wrong, it wasn't today. (P2)

Some people with MCI or dementia expressed a feeling of satisfaction and acceptance with everyday life despite the illness.

Yes it's not funny, it's no fun diagnosis; it is not but it is what it is ... it's so ... everyone must accept their illnesses there are so many ... (P8)

Feelings of gratefulness towards relatives and professionals were expressed, although their help was not always experienced as connected to the dementia disease.

\section{Uncertainty about own ability and environmental reactions}

Uncertainty, both in terms of assessing their own ability and whether relatives noted their difficulties, emerged among people with MCI or dementia. 
No, I don't think so maybe a little [referring to having memory problems]; maybe you should have my wife here. No I don't think she would tell different, but I don't know. (P1)

Some noticed environmental reactions such as being reminded by relatives that "I have told you this several times". Others that relative no longer asked them for important advice.

Our daughter she usually talks to her father, she doesn't think that I understand anything and that feels hard, I mean that makes you feel even worse... (P8).

Not many felt that they could talk about their diagnosis with friends and relatives. It felt taboo.

People don't know how to handle it. If I tell someone about the disease they say; oh dear, and then they don't want to ask anymore...even my sister doesn't ask me about it. (P8)

Some people with MCI or dementia experienced themselves as more open about their shortcomings. Another relative (a daughter) noticed that her mother's close friends were fine with her mother's shortcomings and contacted the relative when making appointments with her, etc.; others distanced themselves and no longer kept in touch. According to the relatives, some people with MCI or dementia were able to hide their shortcomings when socializing with friends. This could sometimes be frustrating for the relatives because the difficulties with coping in everyday life were not understood.

\section{Support in everyday life}

Most people with MCI or dementia did not experience any need for support or assistance in everyday life. They saw it as natural to do things together and that relatives wanted to do certain activities out of their own interest. Many also expressed trust and gratefulness towards their relatives. 
No absolutely not, and if I need any support, I have my wife and for that I'm happy, no, but that's no problem. (P1)

A feeling of being over-protected, however, emerged among people with MCI or dementia.

No, it's him who wants to support me a little (laughing), something like that, I mean I don't want that so much, I don't know but I've never asked him to pick me up earlier. (P6)

Relatives saw things a bit differently and psychosocial support from relatives was common. They struggled to be diplomatic when supporting people with MCI or dementia in everyday life, helping them to stay active and avoid failures.

He washes his clothes himself although I've noticed in the last six month that he uses any programme and mixes up the clothes higgledy-piggledy, but I don't care. As long as he does it, he can do as he likes, because his clothes get washed and that is totally okay. He makes coffee and sometimes it works and sometimes it doesn't; that is the way it is. I mean there are so many things he does and I think that it is good that he does those things, so I like him to keep doing that. (R10)

Feelings of guilt for not being able to help a parent more were experienced.

\section{Support from the health care system}

People with MCI or dementia were relieved that they had come under investigation and were in the health care system. Some negative aspects were however experienced. The way information was presented could give a sense of being seen as an infant and too negative information about the disease could induce feelings of incompetence. A wish for more support in terms of information about the disease and how to handle symptoms was expressed.

Thus, I'm still in a phase where I have not been before. I've had a few knocks, so I have a certain fear, and I don't know what will happen and things like that, this is bothering me a lot ... 
Some people with dementia had experiences from day care services. Both satisfaction and dissatisfaction with the service were experienced. Some had stopped going there. They felt there was a lack of interesting activities and participation and felt that the other participants were sicker than themselves.

The other participants were just old men sitting like this [showing the chin dropped down with open mouth]; there was a guy that was better than me but just one; yeah, it feels a little sad. I mean you can work at a place like that, but then you get the money for it, but to sit on couch like that ... (P10)

I would love to work there but I do not wish to be a patient there. We were not even allowed to help out in the kitchen, and we were served food as if we were idiots! (P7)

Another disadvantage raised regarding day care was the transportation service with waiting times and having to go on long trips to transport other travellers in the same taxi rather than going straight to the home.

\section{Consequences in everyday life for relatives}

Feelings of burden but also sadness for the situation of people with MCI or dementia were common among relatives. The level of burden differed. Not being able to leave the person with MCI or dementia alone was one source of burden. Dissatisfaction with day care could have important consequences as for the spouse who had to quit working when her husband stopped going there. Few felt that they could leave home for more than a day without making special arrangements. Some relatives were able to leave home for a few days and the person with MCI or dementia was able to cope with written notes. A huge balancing act was sometimes required in everyday life and it meant changed roles in the family in many cases. 


\section{Support for relatives}

Having someone to talk to about their difficulties in everyday life was an important kind of support for relatives. This was usually a relative or a friend, but could also be a professional. The fact that the professionals can only be reached at certain times was seen as an obstacle. A fear of being too burdensome to other family members, such as children, was another obstacle. Fear of exposing the person with MCI or dementia also emerged. The relatives who had participated in family education programs, either at the memory clinic or in the community, reported mixed feelings about it. Negative aspects were being much younger or being a relative of people with fewer symptoms than other participants. Positive aspects were being more prepared for the future and having extended information about the disease and knowing where to turn if the situation got worse.

At first when I heard the other stories I thought; is this the future? Then I could see the positive things about it. I am prepared. So it was a bit two-sided. (R11)

Relatives who had not attended any family education programmes wanted advice on how to best act towards the person, how to give the best support in everyday life.

How to act in everyday life, how much should one help. I'm trying anyway. Sometimes if we are just sitting, I say well we don't have any salt here and then I say but you can get it rather than thinking that I have to get it myself. I tell him to get it; such small things. Or if we need more coffee; I say could you serve the coffee, so that I don't do it myself. I'm trying to do so, but I mean this is new for me; he always used to do - ask me do you want more and so on - a complete personality change in many ways. Still, no I don't need any support yet; that may come in the future. (R6)

I don't know if one can stimulate her to take more initiative or not, it's really hard to know how to handle things, because it is not at all what it used to be. (R9) 


\section{Discussion}

The aim of this study was to explore experiences of cognitive impairment, its consequences in everyday life and the need for support felt by people with MCI or mild dementia and their relatives. The extended knowledge provided by this study could improve clinical practice when evaluating patients with suspected dementia and planning for interventions. The main findings were that people with MCI or dementia experienced cognitive changes that could be burdensome, and activity patterns changed. Most considered themselves capable of coping on their own. Participants also expressed feeling safe when being in the health care system and trusting that relatives would stand up for them when necessary. The relatives noticed cognitive changes and disruptions to activities to a greater extent and tried to be supportive in daily life as best they could.

Changed activity patterns covered many different activities in everyday life from work to more personal activities. It was notable that taking care of personal hygiene and appearance was mentioned as changed. This is not included or mentioned as a symptom in the early stages of dementia (Reisberg et al., 1982; Winblad et al., 2004). However, in another qualitative study with the same target group (people with MCI and mild dementia) relatives also reported that some persons neglected their personal appearance, e.g. shaving and washing less frequently (Frank et al., 2006). Normally, a basic ADL measure is not used in clinical investigations in the early stages of the disease and an instrument like the Index of ADL (Katz et al., 1963), which only measures independence or dependence in activities, would probably not have captured these difficulties. The present results, however, show that questions on managing hygiene and more personal activities should be raised at an early stage. The use of an IADL instrument such as the 
Lawton scale (Lawton and Brody, 1969) in clinical settings might be feasible but still there is a chance of missing out on other activities such as leisure or social activities and communication, which seem to deteriorate in the early stages.

There was a discrepancy between the people with MCI and the people with dementia in the way they spontaneously related the consequences in everyday life. It appeared to be easier for those with MCI, while those with dementia needed more prompting and more follow-up questions. A good method of eliciting this information was to go through a normal day and then ask about any shortcomings. This suggests that a check-list or other instrument that covers different areas of activity might be useful when interviewing patients with dementia in clinical settings.

People with cognitive impairment/dementia gradually decrease their areas of activity and their life space (Chung et al., 2008; De Vriendt et al., 2012). An interesting finding in our study was that this was not always experienced as a problem by them, but it was seen as a problem by relatives. The current study also confirmed that many carers of people with dementia express a major loss of social participation (Nay et al., 2014). Carers might have to quit work, give up previous leisure activities or holiday trips and friends may start to avoid them. Finding new contacts could be positive; for example, by connecting with others with similar experiences (Nay et al., 2014). The stigma of Alzheimer disease or dementia is also well known (O'Sullivan et al., 2014) and examples of people who do not know how to act when someone in their vicinity has cognitive impairment and who may even withdraw from further contact were found also in the present study. There is a need for more public information about dementia and the fact that there are different stages and individual differences. People with dementia seem to be dependent 
on relatives and friends who can accept their shortcomings and find ways to handle them.

Having Alzheimer disease is not the same for everyone and the same applies to the carers who have different experiences of the burden felt (Nay et al., 2014). The benefits of carer support groups have been highlighted (Chien et al., 2011); however, in our study it became clear that it is important to have groups that are matched in terms of age and degree of dementia.

There was a discrepancy between the stories of those with MCI and, in particular, those with dementia, and the stories of the relatives. Awareness differed among people with MCI or dementia. Lack of awareness could lead to problems in everyday life according to the relatives and interfered with the use of external memory strategies. In conclusion, experiences of cognitive impairment and its consequences in everyday life were very individual and varied among the participants and their relatives. Thus, it is important for health care professionals to listen to both the people with MCI or dementia and their relatives when planning optimal individual care. People with MCI or dementia and their relatives might need help with how to communicate about these issues and how to handle things in everyday life. In line with earlier studies the present results indicates that even people with MCI may have insight problems (Frank et al., 2006) and need support as well as people with Alzheimer disease (Mårdh et al., 2013).

Important areas for improvement seem to be day care for people in the early stages of the disease, as well as family education for their relatives. There could be problems in clinics getting enough people at a similar phase of the disease at the same time when working with groups. However, this makes it even more important to plan for individual support after the diagnosis is made. Research on experiences from day care and family education programmes is scarce. Some positive experiences have been seen when evaluating day care from the person's point of view 
(Aggarwal et al., 2003; Brataas et al., 2010). This is not totally in line with the results of this study and this area needs further research. Day care has been seen by family caregivers as a means of making everyday life more manageable (Gústafsdóttir, 2014). In this study, the importance of well-functioning day care was illustrated by the spouse who had to give up her work when her husband no longer wanted to go to day care. The fact that people with dementia want more information after having been diagnosed has been seen in earlier research. People with dementia found that the information they were given after diagnosis was aimed at their relatives rather than at themselves (Mountain and Craig, 2012). The results of this study confirm this to some extent even though most of the people with MCI or dementia were not asking for more information. The way information is given is also important. For example, care must be taken to address people with MCI or dementia as competent adults even when the message itself needs to be simplified.

\section{Methodology discussion}

Lincoln and Guba (1985) state that the quality of a study depends on its trustworthiness, criteria of credibility, transferability, dependability and conformability. To enhance the credibility and the dependability of the present study, the data and the interpretation were discussed continuously with the last author. To be able to judge the transferability of the study as a reader, the procedures must be described thoroughly and this has been done. One participant had some minor problems expressing himself, talking in a fragmented way or paraphrasing and sometimes he had difficulties finding his words. Thus, it was an advantage that the interviewer had long experience of people with similar problems and was secure about how to deal with that. This is important for making the participant feel comfortable during the interview. There is a 
risk that the cognitive decline of the participants, even when mild, prevented them from expressing everything they experience in everyday life, due to the nature of the disease. Another approach would have been to combine interviews with observation as in an earlier study (Öhman et al., 2008). However, the aim was to gain a deeper understanding of experiences, and interviews were chosen as the best way to capture this. All the participants had the opportunity to choose the location of the interview and this also enhances the trustworthiness of the study. A weakness of the study is that even though saturation was reached, the sample is rather small and was taken from one clinic. This decreases the generalizability.

\section{Implications for clinicians and future research}

The findings in this study show that living with dementia in the early stages can be burdensome for both those affected by the condition and their relatives. Separate measures for people with MCI or dementia and relatives are needed with regard to impairments in everyday life and the burden felt. Relatives struggle to help and support as much as they can in everyday life. There is room for improvements concerning support from the health care system at the early stages. For example, support or education groups and day care could be tailored more towards the early stages of dementia. Support groups for couples could be an option for some couples. More research is needed on these interventions. The aspect of awareness and how to handle this in everyday life is a great challenge for clinicians and in research. However, this study highlights that when asked about their experiences of cognitive change and support, people with MCI or dementia supply valuable information that may guide treatment planning. 


\section{Conflict of interest declaration}

None.

\section{Description of authors' roles}

M. Johansson designed the study, did the interviews, transcribed the interviews, did the analyses and wrote the paper. J. Marcusson assisted with the design and writing the paper. E. Wressle assisted with the design, analyses and writing the paper.

\section{Acknowledgements}

The authors would like to thank all the participants for sharing their experiences so generously. It is our hope that this will become helpful to people with the same problems or professionals who encounter people with memory problems.

\section{References}

Aggarwal, N., Vass, A. A., Minardi, H. A., Ward, R., Garfield, C. and Cybyk, B. (2003). People with dementia and their relatives: personal experiences of Alzheimer's and of the provision of care. Journal of Psychiatric and Mental Health Nursing, 10, 187-197. doi: 10.1046/j.1365-2850.2003.00550.x.

APA (Ed.) (2004). Diagnostic and Statistical Manual of Mental Disorders. (Fourth Edition, $D S M-I V)$. Washington D.C: American Psychiatric Association.

Avlund, K. and Fromholt, P. (1998). Instrumental activities of daily living: the relationships to self-rated memory and cognitive performance among 75-year-old men and women. Scandinavian Journal of Occupational Therapy, 5, 93-100. 
Barberger-Gateau, P., Commenges, D., Gagnon, M., Letenneur, L. and et al. (1992). Instrumental Activities of Daily Living as a screening tool for cognitive impairment and dementia in elderly community dwellers. Journal of the American Geriatrics Society, 40, $1129-1134$.

Barberger-Gateau, P., Fabrigoule, C., Helmer, C., Rouch, I. and Dartigues, J. F. (1999). Functional impairment in instrumental activities of daily living: an early clinical sign of dementia? Journal of the American Geriatrics Society, 47, 456-462.

Brataas, H. V., Bjugan, H., Wille, T. and Hellzen, O. (2010). Experiences of day care and collaboration among people with mild dementia. Journal of Clinical Nursing, 19, 28392848.

Chien, L. Y., et al. (2011). Caregiver support groups in patients with dementia: A metaanalysis. International Journal of Geriatric Psychiatry, 26, 1089-1098.

Chung, P. Y. F., Ellis-Hill, C. and Coleman, P. G. (2008). Carers perspectives on the activity patterns of people with dementia. Dementia, 7, 359-381.

De Vriendt, P., Gorus, E., Cornelis, E., Velghe, A., Petrovic, M. and Mets, T. (2012). The process of decline in advanced activities of daily living: a qualitative explorative study in mild cognitive impairment. International Psychogeriatrics, 24, 974-986. doi: 10.1007/s12603-012-0381-9.

Delva, F., Edjolo, A., Peres, K., Berr, C., Barberger-Gateau, P. and Dartigues, J. F. (2014). Hierarchical structure of the activities of daily living scale in dementia. The Journal of Nutrition, Health \& Aging, 1-7. doi: 10.1007/s12603-014-0028-0.

Frank, L., et al. (2006). Impact of cognitive impairment on mild dementia patients and mild cognitive impairment patients and their informants. International Psychogeriatrics, 18, 151-162. doi: 10.1017/S1041610205002450 
Gústafsdóttir, M. (2014). The family's experience of sharing the care of a person with dementia with the services in specialized day-care units. Dementia and Geriatric Cognitive Disorders Extra, 4, 344-354.

Katz, S., Ford, A. B., Moskowitz, R. B., Jackson, B. A. and Jaffe, M. W. (1963). Studies of Illness in the Aged, The Index of ADL: A Standardized Measure of Biological and Psychosocial Function. JAMA, 185, 914-919. doi: 10.1001/jama.1963.03060120024016.

Larsson, K. and Thorslund, M. (2006). Old people's health. Scandinavian Journal of Public Health, 34 (Suppl.67), 185-198.

Lawton, M. P. and Brody, E. M. (1969). Assessment of Older People: Self-Maintaining and Instrumental Activities of Daily Living. Gerontologist, 9, 179-186.

Lincoln, Y. and Guba, E. (1985). Naturalistic Inquiry. Newbury Park, CA: Sage Publications.

Mountain, G. A. and Craig, C. L. (2012). What should be in a self-management programme for people with early dementia? Aging \& Mental Health, 1-8. doi: 10.1080/13607863.2011.651430.

Mårdh, S., Karlsson, T. and Marcusson, J. (2013). Aspects of awareness in patients with Alzheimer's disease. International Psychogeriatrics, 25, 1167-1179. doi: $10.1017 / \mathrm{S} 1041610212002335$.

Nay, R., Bauer, M., Fetherstonhaugh, D., Moyle, W., Tarzia, L. and McAuliffe, L. (2014). Social participation and family carers of people living with dementia in Australia. Health \& Social Care in the Community, [Epub ahead of print]. doi: 10.1111/hsc.12163.

O'Sullivan, G., Hocking, C. and Spence, D. (2014). Dementia: The need for attitudinal change. Dementia, 13, 483-497. doi: 10.1177/1471301213478241. 
Pedrosa, H., et al. (2010). Functional evaluation distinguishes MCI patients from healthy elderly people — The ADCS/MCI/ADL scale. Journal of Nutrition, Health \& Aging, 14, 703-709. doi: 10.1007/s12603-010-0102-1.

\section{Petersen, R. C., Caracciolo, B., Brayne, C., Gauthier, S., Jelic, V. and Fratiglioni, L.} (2014). Mild cognitive impairment: a concept in evolution. Journal of Internal Medicine, $275,214-228$.

Qiu, C., Kivipelto, M. and von Strauss, E. (2009). Epidemiology of Alzheimer's disease:occurrence, determinants, and strategies toward intervention. Dialogues in Clinical Neuroscience, 11, 111-128.

Reisberg, B., Ferris, S. H., De Leon, M. and Crook, T. (1982). The Global Deterioration Scale for assessment of primary degererative dementia. American Journal of Psychiatry, $139,1136-1139$.

SBU (2006). Demenssjukdomar (Dementias). SBU Report. Available at http://www.sbu.se/sv/Publicerat/Gul/Demenssjukdomar/.

Strauss, A. and Corbin, J. (1990). Basics of Qualitative Research: Grounded Theory Procedures and Techniques. Newbury Park: CA: Sage Publications.

Wadley, V. G., Okonkwo, O., Crowe, M. and Ross-Meadows, L. A. (2008). Mild cognitive impairment and everyday function: Evidence of reduced speed in performing instrumental activities of daily living. The American Journal of Geriatric Psychiatry, 16, 416-424.

Winblad, B., et al. (2004). Mild cognitive impairment - beyond controversies, towards a consensus: report of the International Working Group on Mild Cognitive Impairment. Journal of Internal Medicine, 256, 240-246. 
Yeh, Y.-C., Lin, K.-N., Chen, W.-T., Lin, C.-Y., Chen, T.-B. and Wang, P.-N. (2011). Functional disability profiles in amnestic mild cognitive impairment. Dementia and Geriatric Cognitive Disorders, 31, 225-232.

Öhman, A., Josephsson, S. and Nygård, L. (2008). Awareness through interaction in everyday occupations: experiences of people with Alzheimer's disease. Scandinavian Journal of Occupational Therapy, 15, 43-51. 
Table 1. Characteristics of the participants

\begin{tabular}{lll}
\hline & MCI $(\mathrm{n}=5)$ & Dementia $(\mathrm{n}=8)$ \\
\hline Participants, men/women & $2 / 3$ & $5 / 3$ \\
Age, mean \pm SD (range) & $72 \pm 4.8(66-77)$ & $70 \pm 7.5(58-78)$ \\
Education: low/middle/high & $1 / 2 / 2$ & $4 / 2 / 2$ \\
Duration of symptoms, years & $2-3$ & $1-7$ \\
Time since diagnoses, years & $0.5-2$ & $1-6$ \\
MMSE score, mean \pm SD (range) & $27 \pm 1(26-28)$ & $22 \pm 2.7(19-26)$ \\
GDS score & 3 & 4 \\
Relatives, men/women & $3 / 2$ & $3 / 5$ \\
Age, mean \pm SD (range) & $71 \pm 4.3(67-77)$ & $63.1 \pm 9.1(47-74)$ \\
Child/spouse & $0 / 5$ & $2 / 6$ \\
\hline
\end{tabular}

MMSE, Mini-Mental State Examination; GDS, Global Deterioration Scale. 